\title{
Die djb-Bundesgeschäftsstelle in Berlin: 10 Jahre nach dem Umzug
}

\section{am 19. März 2012 im Kammergericht, Berlin*}

Unbestreitbar der Höhepunkt meiner bisherigen Amtszeit als Präsidentin des djb war die Feier zum zehnjährigen Bestehen der Bundesgeschäftsstelle in Berlin. In den geschichtsträchtigen Räumen des Kammergerichts und in Gegenwart zahlreicher hochrangiger Vertreter/innen und Repräsentant/inn/en aus Politik und anderen Verbänden hatte ich die besondere Ehre, diese Feier in Anwesenheit meiner Vorgängerinnen im Amt und einiger unserer Ehrenpräsidentinnen mit unseren Mitgliedern und für unseren Verband im vollbesetzten Plenarsaal eröffnen zu dürfen. Dafür gilt mein besonderer Dank den Kolleginnen im djb, und dabei vor allem der Präsidentin des Kammergerichtes Frau Monika Nöhre als der Hausherrin sowie Frau Professorin Dr. Ursula Nelles für ihre fulminante Festrede, die in einem weiten Bogen Rückblick und Ausblick für den djb thematisiert und so humorvoll präsentiert hat. Gefeiert haben wir bei dieser Gelegenheit aber nicht den Ver- band, sondern zehn Jahre Bundesgeschäftsstelle in Berlin. Neben einem Standort und konkreten Räumlichkeiten bedeutet das insbesondere die Präsenz unserer Geschäftsführerin und ihres Teams in der Bundeshauptstadt. Anke Gimbal und ihrer Vorgängerin Martina Bosch sowie allen Mitarbeiterinnen gilt der herzliche Dank der Mitgliedsfrauen im djb für ihre über viele Jahre geleistete engagierte Arbeit für den djb und ihre Identifikation mit den Anliegen des Verbandes, zu deren Verwirklichung sie maßgeblich beigetragen haben und weiterhin mitwirken. Mir persönlich ist es eine große Ehre und besondere Verpflichtung, der Verantwortung gerecht zu werden, die daraus folgt, dass die großartigen Frauen im djb mir das Amt der Präsidentin anvertraut haben. Sehr gerne begleite ich die Geschäftsstelle auf ihrem Weg in das zweite Jahrzehnt.

Ramona Pisal, Präsidentin

\section{Anke Gimbal \\ Bundesgeschäftsführerin, Berlin}

Zehn Jahre war es her, dass die djb-Bundesgeschäftsstelle von Bonn nach Berlin gezogen ist. Anlass genug zu einer Festveranstaltung, die am 19. März 2012 im Kammergericht, Berlin stattfand. Der Sitzungssaal war voll besetzt. Es standen prominente Rednerinnen und Redner auf dem Programm: Dr. Birgit Grundmann, seit November 2009 Staatssekretärin des Bundesministeriums der Justiz, vertrat Bundesjustizministerin Sabine Leutheusser-Schnarrenberger. Als Vertreter der Landes Berlin sprach Senator Thomas Heilmann, seit dem 12. Januar 2012 Senator für Justiz und Verbraucherschutz. Hausherrin Monika Nöhre, Präsidentin des Kammergerichts seit 2002, ist der djb zu großem Dank verpflichtet, da sie den großen Sitzungssaal für die Feier zu Verfügung stellte. Festrednerin Prof. Dr. Ursula Nelles leitet seit dem 1. Oktober 2006 als Rektorin die Westfälische Wilhelms-Universität in Münster. 2004 wurde sie dort zur Dekanin der Juristischen Fakultät gewählt. Mit ihr stand 2006 zum zweiten Mal eine Frau an der Spitze der Universität Münster. Als letztes sprach Juliane Kirchner, Fachanwältin für Strafrecht.

Die Vorträge befassten sich vor allem mit der Bundesgeschäftsstelle des djb, ihrem Umzug 2001 von Bonn nach Berlin sowie den Umzugsmodalitäten. Ramona Pisal stellte in groben Zügen die Entwicklung der Geschäftsstelle dar. 1948 in Dortmund gegründet, bekam der Verband seine erste Verwaltungszentrale in der Person von Dr. Hertha Engelbrecht (1922-2012), die 1985 nach ihrer Pensionierung ehrenamtlich die Geschäftsführung des djb übernahm. 1992 wurde ein Ein-Raum-Büro in Bonn daraus. Martina Bosch wurde 1994 zur Mitgeschäftsführerin ernannt und die Geschäftsstelle ent- wickelte sich dynamisch und ab 1996 in repräsentablen Räumen zu einem Betrieb, in dem bis zu vier Frauen arbeiteten. Ab 1997 und für die folgenden fünf Jahre leitete Martina Bosch die Geschäftsstelle.

Nachdem Berlin die neue deutsche Hauptstadt wurde und der Bundestag seinen Umzug beschloss, begann im djb wie in vielen Verbänden die Diskussion des Für und Wider eines Umzugs. Sie wurde in der Amtszeit der 1. Vorsitzenden Prof. Dr. Ursula Nelles wie bei den allermeisten Verbänden schließlich zugunsten des Umzugs nach Berlin entschieden. Prof. Dr. Ursula Nelles kommentierte dies mit den Worten: „Ich will nun nicht die heftigen und mit zunehmender Uhrzeit zunehmend ernüchternden Sachdebatten nachzeichnen, die der Vorstand damals geführt hat, noch die nicht minder vehemente Diskussion auf dem 33. Bundeskongress. Vielmehr möchte ich aus der Retrospektive beleuchten, was meines Erachtens gut gelaufen ist und die Richtigkeit der Entscheidung, die Geschäftsstelle nach Berlin zu verlagern, untermauert." Ramona Pisal stimmte dem zu: „Der djb arbeitet frauenpolitisch. Wir sind Lobbyistinnen für die Gleichstellung der Frauen. Wir wollen Einfluss nehmen, gehört werden. Politik wird da gemacht, wo die Regierung sitzt. Das war in Bonn so, und das würde nun in Berlin so sein. Um mit unserer Kanzlerin zu sprechen: Die Maßnahme war mit Kosten verbunden, aber sie war alternativlos. “ Juliane Kirchner drückte es so aus: „Wäre der djb in Bonn geblieben, hätte er seinen Wirkungsbereich verloren. Die Karawane ist weitergezogen und wenn man die Leittiere in seine Richtung bewegen will, darf man nicht hinter der Herde zurückbleiben. Das ist nicht weiter überraschend. “

\footnotetext{
Die Veranstaltung wird auf der djb-Homepage dokumentiert: http://www.djb.de/Veranstaltungen/

Veranstaltungsdokumentationen/2012Berlin1o/
} 


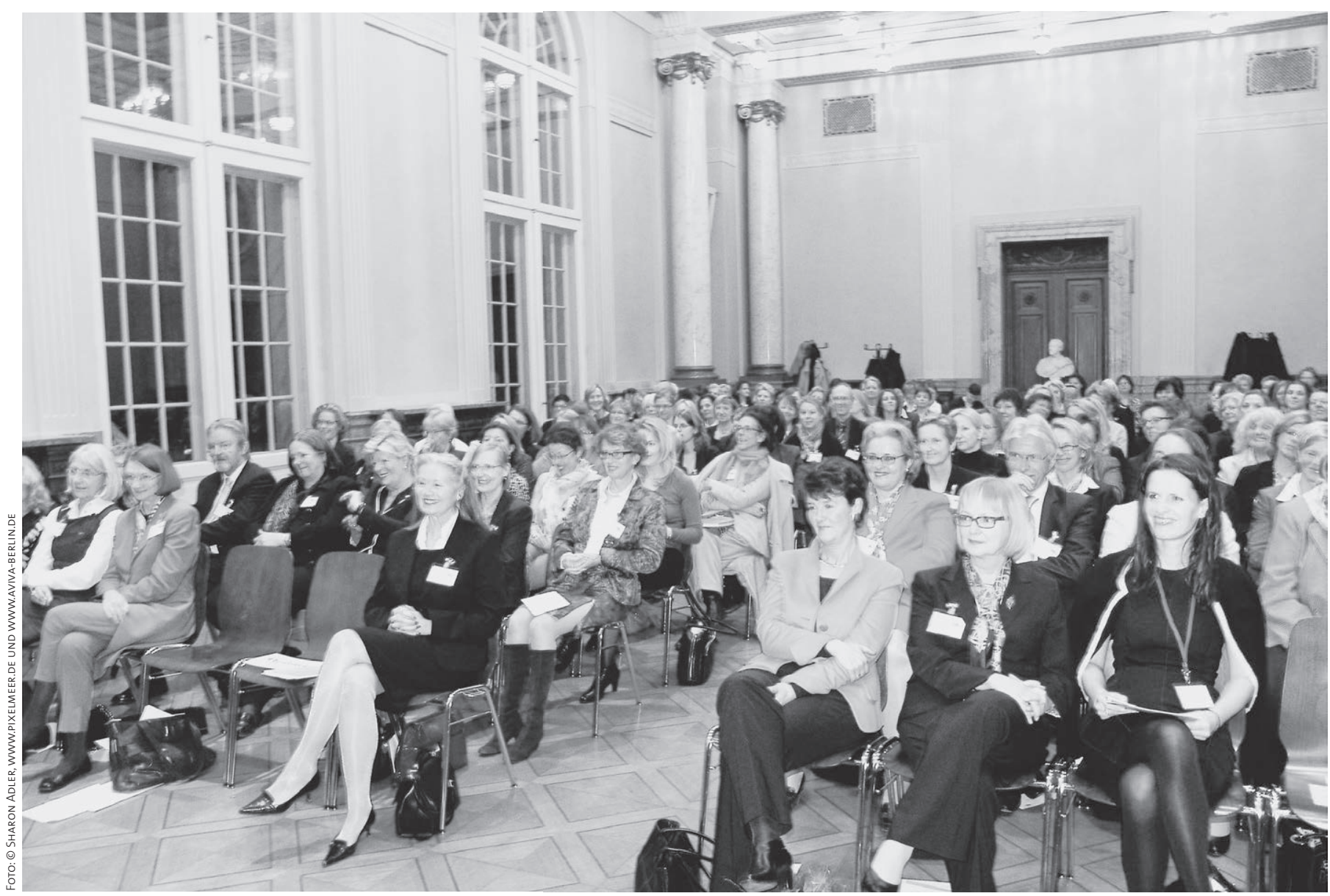

$\Delta$ Der geschichtsträchtige Sitzungssaal des Kammergerichts an der Elßholzstraße ist voll besetzt. Wie Kammergerichtspräsidentin Monika Nöhre in ihrer Begrüßungsrede als Hausherrin schilderte: Von August 1944 bis Januar 1945 fanden in diesem Raum unter dem Vorsitz von Roland Freisler mehrere Schauprozesse gegen die Beteiligten am Attentat auf Hitler im Juli 1944 statt. Nach Kriegsende 1945 wurde das Gebäude Sitz des Alliierten Kontrollrates für Deutschland. Dass das Kammergericht zu Beginn des 20. Jahrhunderts der Wohnort von Elisabeth Heinroth war, ist eine ganze andere Geschichte. Als Elisabeth Rindfleisch, geschiedene Bosch, heiratete sie den Juristen Wilhelm Heinroth, der 1909 zum Präsidenten des Berliner Kammergerichts berufen wurde. 1913 war das neue Gebäude fertig und die Familie zog dort ein. Unter dem Pseudonym Klaus Rittland veröffentlichte sie bis zu ihrem Tod 192017 Bücher, zumeist gesellschaftskritische Unterhaltungsromane.

Margret Diwell trat sodann 2001 ihr Amt als djb-Präsidentin an, um als erstes und sehr erfolgreich den eigentlichen Umzug durchzuführen. Die Geschäftsstelle in der Anklamer Straße 38 in Berlin-Mitte wurde am 8. März 2002 in Gegenwart vieler Gäste aus der Politik, der Justiz, den Verbänden, den Medien und vor allem auch vieler djb-Mitglieder offiziell eröffnet. Der djb hatte mit Anke Gimbal nun die erste Berliner Geschäftsführerin. Die nun vergangenen zehn Berliner Jahre sahen eine große Zahl djb-Veranstaltungen. Gestiegen sind die Zahlen der Stellungnahmen und Pressemitteilungen. Die Verbandszeitschrift bekam ein neues und immer professionelleres Gesicht. Bücher, Zeitschriften und andere Beiträge wurden herausgegeben - und vorher geschrieben - wie zum Beispiel 2005 „Juristinnen - Lexikon zu Leben und Werk“ von Dr. Marion Röwekamp. Eine Ausstellung „Juristinnen in der DDR “ wurde maßgeblich von Prof. Dr. Rosemarie Will und Dr. Marion Röwekamp erstellt, 2011 in Potsdam eröffnet und auch am 19. März 2012 im Kammergericht präsentiert. Zweimal - 2009 für eine herausragende Dissertation und 2011 für eine Habilitationsschrift - wurde der Marie Elisabeth Lüders-Wissenschaftspreis des djb verliehen. Im Konferenzraum der Geschäftsstelle finden die Bundesvorstands- und die meisten Kommissionssitzungen statt. Genauso ist sie der Ort der Wahl für weitere Gespräche mit Regierungs-, Verbands- und sonstigen Vertreterinnen und Vertretern. Häufig wird der djb von Gruppen ausländischer Juristinnen besucht und zum Girls Day kommen gerne auch Schülerinnen. Weitere Aktivitäten wie das vom BMFSFJ geförderte Projekt „Aktionärinnen fordern Gleichberechtigung“ werden in Berlin koordiniert.

$\mathrm{Zu}$ den aktuellen Projekten gehört auch die von Vizepräsidentin Eva Schübel initiierte Aktion „Frauen in die Roten Roben“. Staatssekretärin Dr. Birgit Grundmann äußerte dazu: „Diese Tendenz, hoher weiblicher Anteil in der Masse aber geringe Repräsentanz in der Spitze, findet sich leider auch in der Justiz. Während sich der Prozentsatz etwa der Proberichterinnen wahrlich sehen lassen kann, sieht es bei den Beförderungsstellen längst nicht so rosig aus. Dass bei der letzten Bundesrichterwahl im Jahr 2011 von den 23 Wahlvorschlägen für den BGH nur drei Frauen waren, ist ein trauriger Beleg dafür. $\mathrm{Da}$ ist es schon fast als Erfolg zu werten, dass unter den vier 
Gewählten wenigstens eine Frau gewesen ist. Hier kann das BMJ nicht regulierend eingreifen. Denn es sind die Justizminister der Länder - einer sitzt ja heute hier -, die als Mitglieder des Richterwahlausschusses diesem Gremium die Vielzahl der Personalvorschläge vorlegen. Aber wir als BMJ können appellieren, hier mehr Frauen vorzuschlagen. Und das hat die Ministerin im Vorfeld der jetzt anstehenden Bundesrichterwahl in mehreren sehr deutlichen Schreiben auch getan. (...) um es klar zu sagen: Es mangelt nicht an qualifizierten und interessierten Kandidatinnen, die zu einer Justizkarriere bereit sind. Es bedarf aber gezielten politischen Drucks, um die gläserne Decke zu durchschlagen, die sich über dem Gros der Juristinnen immer noch aufspannt. Einen Stein ins männlich besetzte Glashaus hat Ihre Initiative „Frauen in die Roten Roben“ geworfen und im Dach einige Risse hinterlassen. Diese werden vermutlich - hoffentlich! - bei der nächsten Bundesrichterwahl in zehn Tagen sichtbar werden. Details kann ich Ihnen natürlich nicht nennen. Ich erwarte aber, dass unsere gemeinsamen Bemühungen nicht folgenlos bleiben.“

Die Menge der Arbeit steigt für haupt- und für ehrenamtlich Tätige, der rechtspolitische Einfluss auch. Die gegenwärtige djb-Präsidentin Ramona Pisal: „Welcher Art die Herausforderungen technischer oder sonstiger Natur auch sein werden: Wir werden sie zuversichtlich in Angriff nehmen und bewältigen, jede an ihrem Platz.“

Doch die Konzentration auf Berlin hat auch eine andere Seite. Dazu merkte Prof. Dr. Ursula Nelles: an: „Ich beobachte jedenfalls - anhand der aktuellen informationen -, dass Berichte aus Landes- und Regionalgruppen dürftiger und die dortigen Angebote stereotyper werden. Ich beobachte, dass die große



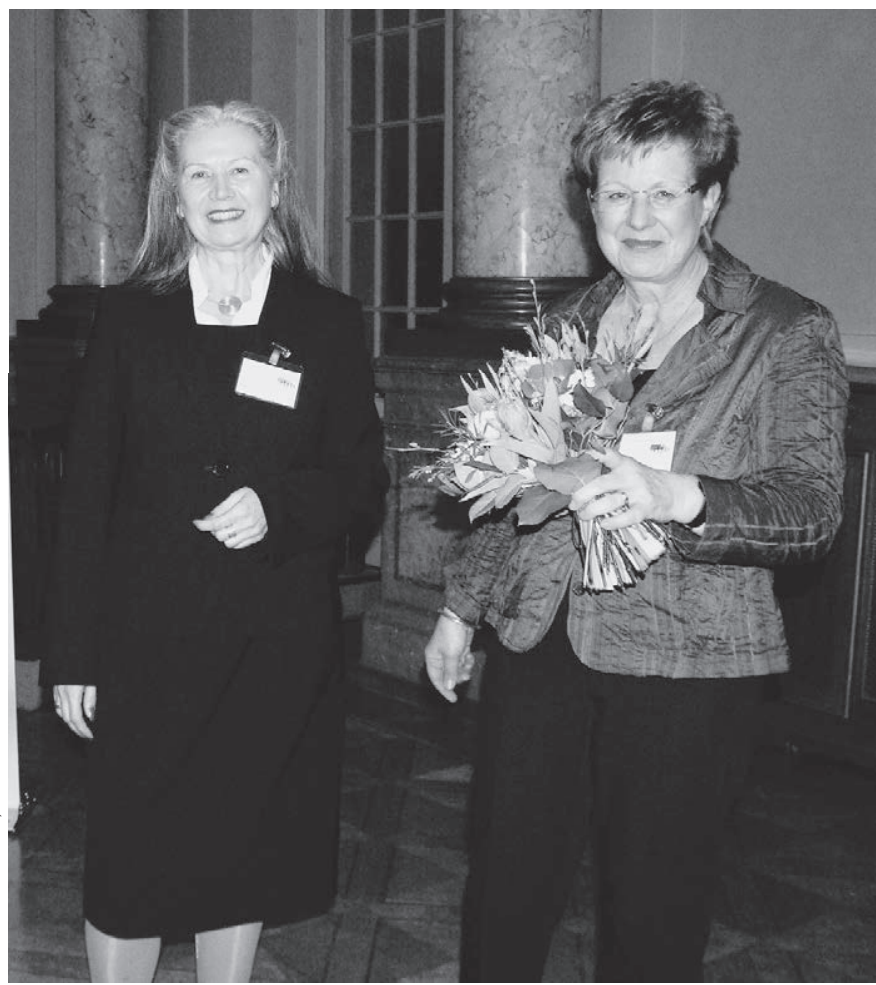

$\Delta$ Präsidentin des djb, Ramona Pisal (links), hebt die Leistungen von Rechtsanwältin Martina Bosch, Geschäftsführerin des djb bis 2001 in Bonn hervor und dankt für ihr überobligatorisches Engagement in ihren zehn Jahren hauptamtlicher Verbandsarbeit. Seitdem ist sie weiter - nun ehrenamtlich - aktiv, insbesondere als Vorsitzende der Regionalgruppe Bonn von 2005 bis 2009 und als Vorstandsmitglied im Regionalgruppenbeirat von 2006 bis 2011. Seit 2005 arbeitet sie außerdem an dem Aufbau und der Vervollständigung des online-Archivs des djb.

$\varangle$ Grußworte sprachen die Staatssekretärin im Bundesjustizministerium Dr. Birgit Grundmann und der Senator für Justiz und Verbraucherschutz des Landes Berlin Thomas Heilmann. Dr. Birgit Grundmann vertrat Bundesjustizministerin Sabine Leutheusser-Schnarrenberger, die kurzfristig zur Europäischen Kommission nach Brüssel reisen musste. Dr. Grundmann ist seit November 2009 Staatssekretärin des Bundesministeriums der Justiz. Bis 1993 war sie als Rechtsanwältin tätig, u.a. in einer Hamburger Kanzlei und auch bei Field Fisher Waterhouse in London. Seitdem ist sie in verschiedenen Funktionen im und für das Bundesministerium der Justiz unterwegs. Ihre juristischen und rechtspolitischen Schwerpunkte sind gewerblicher Rechtsschutz, Kartell- und Wettbewerbsrecht, später Mietrecht sowie Familien-, Erb- und Unterhaltsrecht. Senator Thomas Heilmann ist Volljurist und seit dem 12. Januar 2012 Berliner Senator für Justiz und Verbraucherschutz. Zuvor war er in der Privatwirtschaft tätig, und zwar als Gründer der Werbeagentur Delta-Design, die 1991 in „Scholz \& Friends“ aufging. Bis 2001 war er deren geschäftsführender Gesellschafter in der Niederlassung Berlin. Anschließend und bis 2008 war er deren Vorstandsvorsitzender. Seit 1980 ist Thomas Heilmann Mitglied der CDU, seit 2009 stellvertretender Vorsitzender des CDULandesverbandes Berlin. Er äußerte sich in seinem Grußwort ausführlich zu dem in Deutschland herrschenden Mangel an Frauen in Führungspositionen der Wirtschaft und sprach sich klar für eine rigorose Quote für Aufsichtsräte aus, da es ohne einen solchen Anstoß viel zu langsam voran gehen würde mit der Wirtschaft. Das sei nicht nur eine Frage der Gerechtigkeit, sondern auch eine Frage des Überlebens der deutschen Wirtschaft. 


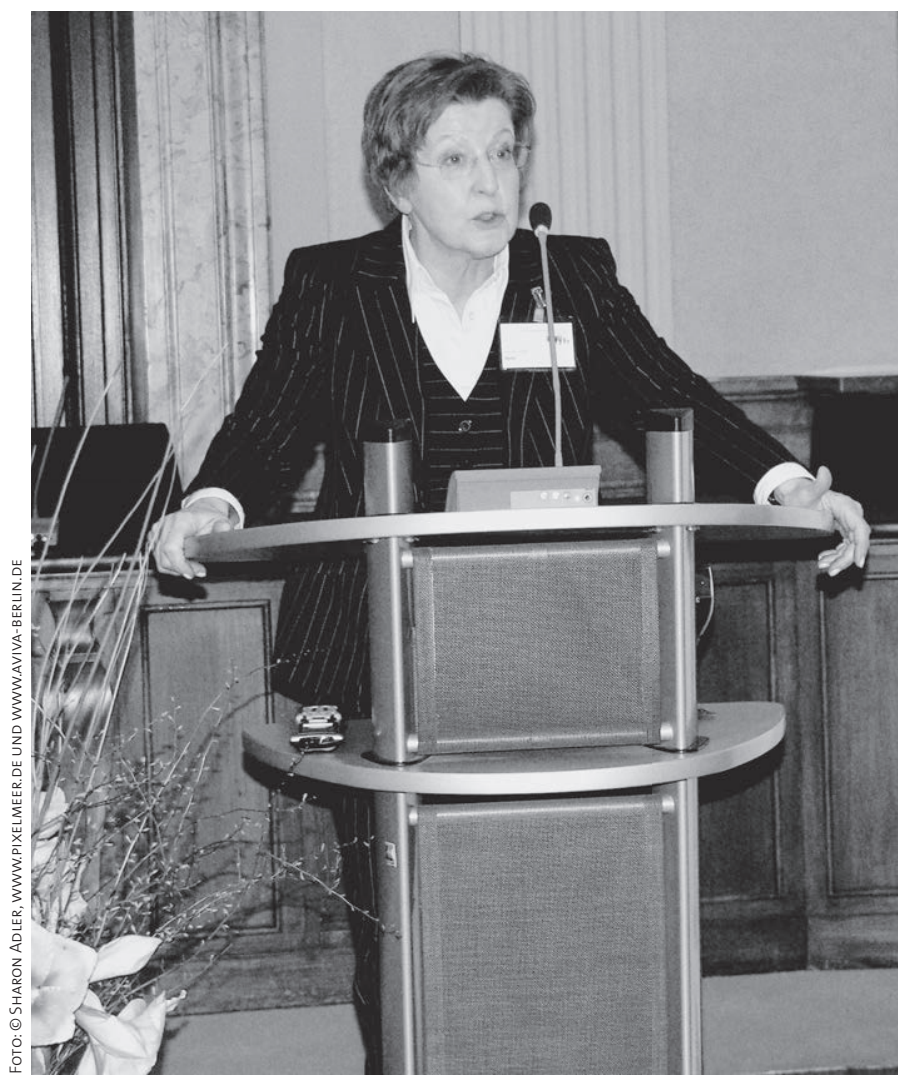

und schöne „Berliner Landesgruppe“ und „der djb“ in der Außenwirkung stärker verschmelzen. Das ist gut: Stichwort Synergie. Für die Binnenkommunikation könnte dieser Effekt freilich gefährlich werden: Die Basis des djb sind noch immer die einzelnen Mitglieder; wir sind kein Dachverband, sondern ein Verein; die Mitgliedschaft im djb muss für Juristinnen in ganz Deutschland attraktiv werden. Das gilt umso mehr, als zweifellos noch vorhandene, strukturelle Diskriminierungen den jungen Frauen

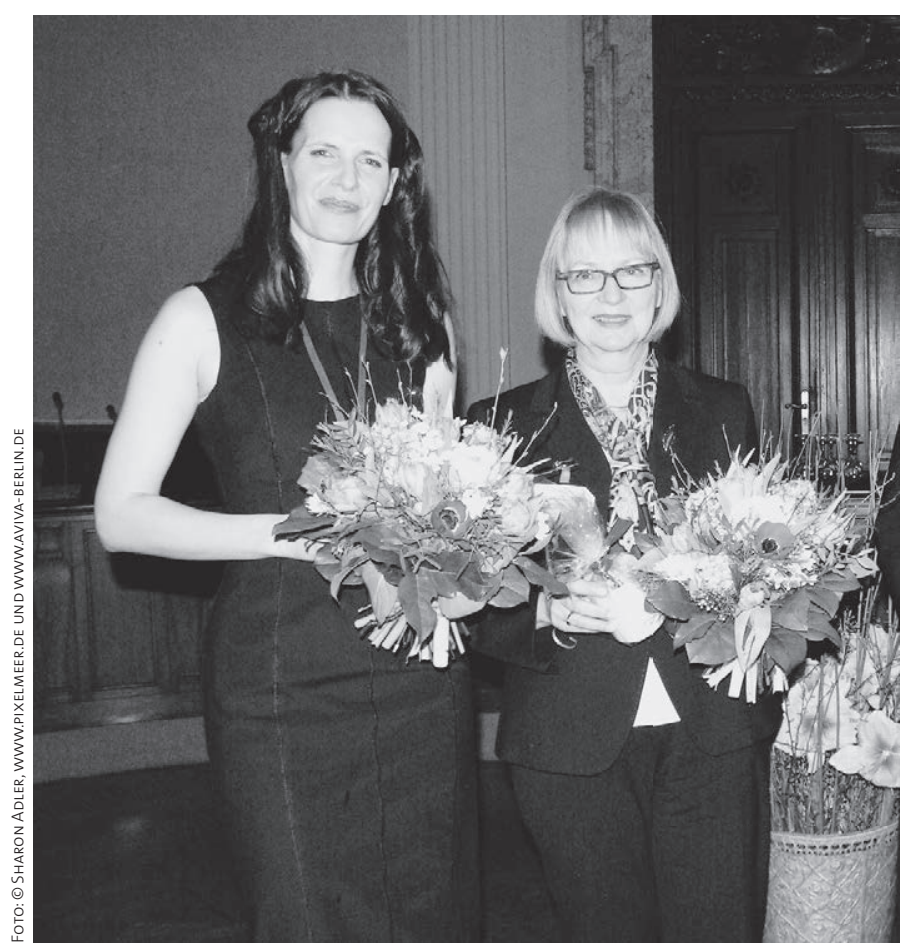

$\triangleleft$ Prof. Dr. Ursula Nelles, als 1. Vorsitzende des djb von 1997 bis 2001 zuständig für den Umzug der Geschäftsstelle von Bonn nach Berlin, hält die Festrede. Sie ist seit dem 1. Oktober 2006 Rektorin der Westfälischen Wilhelms-Universität in Münster. 1990 in Münster habilitiert, erhielt sie nach ihrer Zulassung als Rechtsanwältin Lehraufträge in Münster und Hamburg sowie eine Gastdozentur an der Katholischen Universität Nijmegen in den Niederlanden. Es folgte eine Professur für Strafrecht und Strafprozessrecht an der Universität Bremen sowie Lehraufträge an den Universitäten Greifswald und Düsseldorf. 1994 kehrte sie zur Westfälischen Wilhelms-Universität nach Münster zurück und erhielt an der dortigen Juristischen Fakultät eine Professur für Strafrecht, Strafprozessrecht und Wirtschaftsstrafrecht. Gleichzeitig wurde sie Direktorin des Instituts für Kriminalwissenschaften. 2004 wurde sie zur Dekanin der Juristischen Fakultät der Westfälischen WilhelmsUniversität gewählt. Mit Ursula Nelles stand 2006 zum zweiten Mal eine Frau an der Spitze der Universität Münster, die dann auch die erste Universität mit weiblicher Doppelspitze war - mit Kanzlerin und Rektorin. Als ob das nicht genug gewesen wäre (ist es auch nicht), schlug Ursula Nelles noch eine Prorektorin vor, die einstimmig gewählt wurde und die Universität Münster zur ersten Universität mit einen paritätisch besetzten Rektorat - drei Frauen, drei Männer - machte. Sie war Gründungsvorsitzende des Europäischen Juristinnenbundes (EWLA). Seit 1990 wirkt sie für den djb regelmäßig als Expertin zu strafrechtlichen Fragestellungen in zahlreichen Anhörungen vor Ausschüssen des Deutschen Bundestages mit. Auch die Reform der Verbandsstruktur des djb wurde während ihrer Amtszeit in Angriff genommen.

oft nicht mehr - oder besser: noch nicht - auffallen. Wir riskieren, dass die nächste Generation von Frauen ihre Wachsamkeit verliert und der Backlash sie deshalb unvorbereitet und umso verheerender treffen könnte. “ Und sie warnte „Konzentration darf nicht mit Zentralisierung verwechselt werden oder damit zu stark einhergehen. Vielmehr setzt eine Konzentration das Vorhandensein einer breiten Basis voraus.“

Es bleibt also immer etwas zu tun.

$\checkmark$ Zwei der Rednerinnen: Juliane Kirchner (links) und Monika Nöhre. Monika Nöhre begrüßte die Veranstaltungsteilnehmer/innen als Hausherrin. Sie ist seit 2002 Kammergerichtspräsidentin und Expertin für Fragen der Justizreform. Von 1994 bis 2000 war sie Leiterin des Amtes für Allgemeine Verwaltung in der Hamburger Justizbehörde und zugleich Leiterin des Projektes „Justiz 2000“, das die Maßnahmen zur Modernisierung der Hamburger Justiz bündelte. Vor dem Beginn ihrer Tätigkeit als Richterin hatte sie auch als Rechtsanwältin gearbeitet. Im März 2000 wurde sie Vizepräsidentin des Hanseatischen Oberlandesgerichts. Die damalige Justizsenatorin Karin Schubert erklärte sie anlässlich ihrer Wahl durch das Berliner Abgeordnetenhaus 2002 zu einem „Glücksfall für die Berliner Justiz“. Juliane Kirchner ist seit 2004 Rechtsanwältin in einer mittelständischen Kanzlei auf dem Gebiet des Strafrechts. Fachanwältin für Strafrecht ist sie seit 2007. Seit 2009 arbeitet sie in der Kanzlei Dr. Hille \& Partner in Augsburg. Ihre Spezialgebiete sind Kapitalstrafsachen, Betäubungsmittelstrafsachen und Präventivberatung Sie hält sehr gerne und viele Vorträge - deswegen sagte sie die Rede zum Umzugsjubiläum des djb gerne zu - und erhält dafür auch Preise. Ihre „Jura macht glücklich“-Rede wurde mit dem 1. Preis beim Rednerwettstreit des DAV 2006 bedacht. Ihr „Plädoyer für die Freiheit“ erhielt den 1. Preis beim Rednerwettstreit des DAV 2008. Dazu passt: sie war Moderatorin beim lokalen Radiosender Fantasy. 


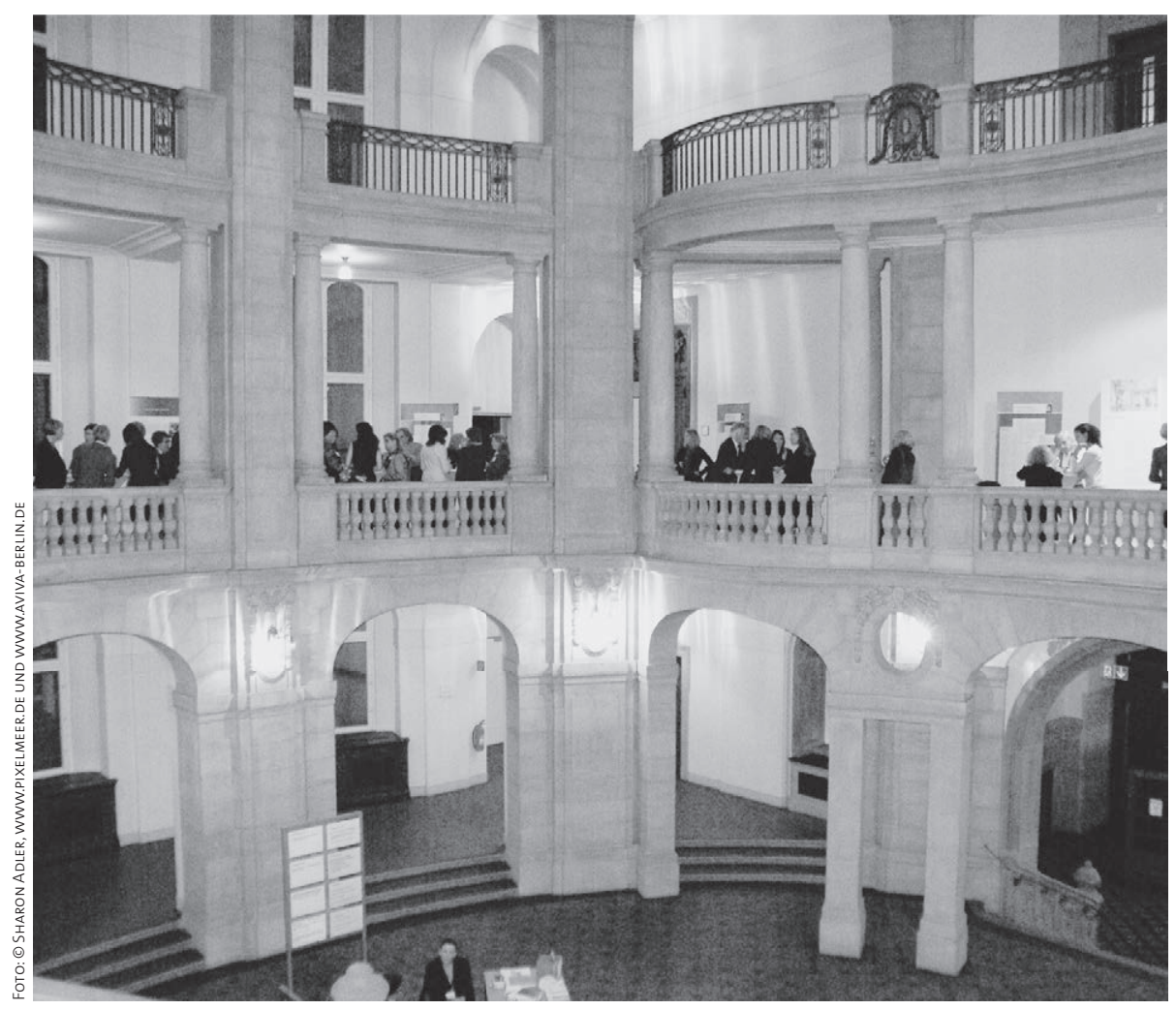

$\checkmark$ Das Kammergericht bietet einen großartigen Rahmen für den Empfang. Im Hintergrund ist die Wanderausstellung des djb „Juristinnen in der DDR“ zu sehen, die 2011 im Landgericht Potsdam eröffnet wurde. Die 23 Ausstellungstafeln befassen sich mit dem Status des Rechts in der DDR, der Rolle der Juristinnen und Juristen im DDRRechtssystem, der juristischen Ausbildung usw. Die weiteren Tafeln porträtieren zwölf Juristinnen aus drei Generationen: (1) der Generation mit bürgerlichem Hintergrund, (2) der DDR-Generation und (3) der Generation mit geteiltem Berufsleben in der DDR und im wiedervereinten Deutschland. Die Ausstellung kann von den Landesverbänden und Regionalgruppen des djb ausgeliehen werden, aber auch von anderen geeigneten öffentlichen und privaten Institutionen wie Gerichten und Ministerien, die sich mit dem Thema Juristinnen und DDR auseinandersetzen möchten. Sie besteht aus 23 farbigen Roll-ups $(215 \times 85 \mathrm{~cm})$ und kann ohne großen technischen Aufwand präsentiert werden.

\section{Sitzung des Regionalgruppenbeirates vom 20. bis 22. April 2012 in Bremerhaven}

\section{Andrea Kirberger \\ Vorstand Regionalgruppenbeirat, Stellvertretende Vorsitzende der RG Dresden; Rechtsanwältin, Fachanwältin für Bau- und Ar- chitektenrecht, Dresden}

Der Regionalgruppenbeirat (RGB) des djb tagt einmal jährlich mit dem Ziel des Informations- und Erfahrungsaustausches zwischen den Regionalgruppen und Landesverbänden. In diesem Jahr kamen vom 20. bis 22. April 2012 insgesamt 22 Delegierte aus ganz Deutschland in Bremerhaven zusammen. Dabei scheuten die Teilnehmerinnen keinen zeitlichen Aufwand, sogar aus Konstanz und aus Brüssel waren Vertreterinnen angereist.

Zum Auftakt der Sitzung am Samstagvormittag ließ es sich der Präsident des Amtsgericht Bremerhaven, Uwe Lissau, der freundlicherweise einen Sitzungssaal im Amtsgericht für die Tagung zur Verfügung gestellt hatte, nicht nehmen, ein Grußwort an die angereisten Teilnehmerinnen zu richten.

Anschließend berichtete die Präsidentin des djb, Ramona Pisal, über die Arbeit des djb auf Bundesebene und insbesondere ihre Tätigkeit seit dem Bundeskongress in Potsdam, auf dem sie bei der Mitgliederversammlung zur neuen Präsidentin gewählt worden war. Die Teilnehmerinnen waren beeindruckt von der Vielzahl der wahrgenommenen Termine und geknüpf- ten bzw. aufgefrischten Kontakte. Mit großem Interesse sind auch die Ausführungen von Ramona Pisal zur Aktion „Frauen in die Roten Roben“, die von der Vizepräsidentin des djb Eva Schübel initiiert worden ist, sowie zu dem nunmehr schon im dritten Jahr laufenden Projekt „Aktionärinnen fordern Gleichberechtigung “ aufgenommen worden. Ramona Pisal verwies ergänzend auf die „Berliner Erklärung“ mit der Bitte, diese unter www.berlinererklaerung.de zu unterstützen.

Bundesgeschäftsführerin Anke Gimbal gab einen Überblick über die geplanten Veranstaltungen, so insbesondere den djbEmpfang beim Deutschen Juristentag am 19. September 2012 sowie den nächsten Bundeskongress in Leipzig im September 2013. Sie erinnerte darüber hinaus noch einmal an die im Rahmen des Kongresses in Potsdam im vergangenen Jahr eröffnete Wanderausstellung „Juristinnen in der DDR “, die von den Regionalgruppen und Landesverbänden präsentiert werden kann.

Anne Brozat, Vertreterin der Jungen Juristinnen im Bundesvorstand, erläuterte das von ihr und ihrer Kollegin Katharina König entwickelte Mentoring-Programm, in dessen Rahmen von Ursula Matthiessen-Kreuder Telefonkonferenzen zum Thema Karriereplanung und Berufsorientierung geleitet werden. Katharina König und Anne Brozat haben darüber hinaus bereits konkrete Ideen, wie das Mentoring-Programm ausgeweitet werden kann. 